\title{
SÍNDROME DEL BURNOUT Y ENGAGEMENT EN PROFESIONALES DEL SISTEMA PENITENCIARIO COSTARRICENSE ${ }^{1}$
}

\section{BURNOUT AND ENGAGEMENT IN PROFESSIONALS OF COSTA RICAN PRISION SYSTEM}

\author{
Manuel Antonio Molina Brenes* \\ Marjorie Moreno Salas**
}

RESUMEN

El Síndrome del Burnout se define como una condición psicológica de agotamiento a nivel personal, cinismo e ineficacia profesional, que puede presentarse en profesionales que laboran directamente con seres humanos, mientras el Engagement como constructo opuesto, es una condición caracterizada por el vigor, la dedicación y la absorción. Las variables anteriores fueron exploradas en 180 profesionales, colaboradores del Sistema Penitenciario costarricense. La técnica de recolección de información fue la encuesta y el muestreo por conveniencia. Los resultados de un cuestionario de 49 preguntas, indicaron la presencia de un bajo nivel de Burnout ( $\mathrm{n}=10)$ y un alto nivel de Engagement $(\mathrm{n}=170)$, una media de 42,04 horas de trabajo semanal y un promedio de 8,59 horas semanales dedicadas a la recreación. Además, el 44,4\% de los(as) participantes estarían dispuestos a cambiar de trabajo. Se concluye una relación negativa y estadísticamente significativa entre el Burnout y el Engagement, junto con una adecuada confiablidad en la medición, también se debe priorizar la investigación de variables asociadas como el agotamiento, la recreación y el uso del tiempo libre.

PALABRAS CLAVE: SÍNDROME DEL BURNOUT * ENGAGEMENT * SISTEMA PENITENCIARIO * TRABAJADORES PROFESIONALES * CIENCIAS SOCIALES * CONDICIONES DE TRABAJO

$1 \quad$ Este estudio fue posible gracias al esfuerzo de la Universidad Católica de Costa Rica y de la Dirección General de Adaptación Social del Ministerio de Justicia y Paz. El equipo de investigación agradece a los(as) funcionarios(as) que colaboraron con la investigación completando el cuestionario proporcionado.

Ministerio de Justicia y Paz (CR) y Escuela de Psicología, Universidad de Costa Rica (UCR).

manuelmolinab@gmail.com

** $\quad$ Escuela de Psicología y Centro de Investigaciones Psicológicas Avanzadas (CIPA), Universidad Católica de Costa Rica.

maryyuri12@gmail.com 


\title{
ABSTRACT
}

\begin{abstract}
Burnout Syndrome is defined as a psychological condition of exhaustion at the personal, professional cynicism and inefficiency that can occur in professionals working directly with humans, while the opposite construct, the Engagement is a condition characterized by vigor, dedication and absorption. The above variables were explored in 180 professional, all of them, collaborators of Costa Rican prison system. The technique of data collection was the survey and sampling of convenience. The results of a questionnaire of 49 questions, indicated the presence of a low level of Burnout $(n=10)$ and a high level of Engagement $(n=170)$, an average of 42,04 hours worked per week and an average of 8,59 hours a week devoted to recreation. In addition, $44,4 \%$ of the participants would be willing to change jobs. We conclude a statistically significant negative relationship between Burnout and Engagement, together with adequate reliable measurement of the variables, also you should prioritize the investigation of variables with Burnout, recreation and leisure use.
\end{abstract}

KEYWORDS: BURNOUT SYNDROME * ENGAGEMENT * PENITENTIARY SYSTEM * PROFESSIONAL WORKERS * SOCIAL SCIENCES * WORKING CONDITIONS

\section{INTRODUCCIÓN}

La Dirección General de Adaptación Social del Ministerio de Justicia y Paz es la institución costarricense encargada de la custodia $y$ el tratamiento de personas indiciadas $y$ sentenciadas, debido a la presunta comisión o la comisión de algún hecho delictivo (Ley 4762, mayo 1971).

En un grupo de profesionales, se exploró la presencia del Síndrome del Burnout, también conocido como el Síndrome del Quemado, el Síndrome del Fundido o el Síndrome de quemarse por el trabajo, denominaciones consideradas sinónimos; al mismo tiempo, se valoró el Engagement, constructo teórico opuesto al primero. El Síndrome del Burnout, se define como una respuesta prolongada a estresores crónicos a nivel personal y relacional en el trabajo, determinado a partir de tres dimensiones: agotamiento emocional, cinismo e ineficacia profesional. El Engagement se asocia a un estado cognitivo-afectivo de bienestar persistente, asociado con vigor, dedicación y absorción (Breso, 2007).

El estudio se llevó a cabo con un grupo de profesionales de ciencias sociales, quienes laboran directamente con "privados y privadas de libertad": personas recluidas en centros penitenciarios o en la modalidad abierta, ya sea del programa institucional, semi-institucional; programa de atención en comunidad y el programa de atención a la población penal juvenil.

El Síndrome del Fundido o Burnout se asocia principalmente con proveedores de cuidados específicos o servicios especializados (Freudenberger, 1974; Maslach, 1976; Maslach y Jackson, 1986; Pines y Aronson, 1988 citados por Fidalgo, 2006), quienes laboran brindando atención directa a otras personas; dentro de estos profesionales se ubican especialmente educadores y personal de salud.

Fue de interés trabajar con profesionales de la administración penitenciaria, debido a la importancia de indagar aspectos emocionales, afectivos y contar con datos empíricos sobre esta población. Además, se asumió como una premisa que laborar en esta institución y permanecer en contacto con la población mencionada implica responder a demandas como: trabajar en una institución total y las consecuencias asociadas. Dicho espacio se definió "como un lugar de residencia y trabajo, donde un gran número de individuos en igual situación, aislados de la sociedad por un período apreciable de tiempo, comparten en su encierro una rutina diaria administrada formalmente" (Goffman, 1971: 13) y el sistema penitenciario constituye un ejemplo de estos espacios. El manejar las demandas de la población privada 
de libertad, se reconoce como una labor eventualmente agotadora y extenuante a nivel físico $y$ emocional.

El objetivo de la presente investigación fue caracterizar la presencia de este síndrome y la condición opuesta (Engagement) en funcionarios(as) de dicha institución. Al mismo tiempo, interesó explorar otras variables laborales, por ejemplo, la antigüedad, el uso del tiempo libre, el tiempo disponible para realizar las tareas asignadas, entre otras.

Desempeñar un empleo con las condiciones mínimas, que contribuya a mejorar el nivel de salud $y$ promueva el crecimiento integral, es un derecho humano. Sin embargo, es conocida la carencia de servicios en salud mental en las instituciones estatales, así como, la ausencia de políticas consistentes $y$ sostenidas para la adecuada gestión del talento humano.

En cuanto a los antecedentes nacionales, se registran abundantes estudios con personal de salud, algunas de las investigaciones fueron desarrolladas por: Porras (1997), Bianchini (1997) y Madrigal (2006), quienes en sus aportes presentan desde discusiones sobre la conceptualización del Síndrome, hasta datos estadísticos con diferentes poblaciones; pero no constituyen un antecedente directo del presente estudio.

Un aporte latinoamericano (Chile, Argentina y Costa Rica, entre otros) en profesionales de la salud es el de Grau, Flichtendrei, Suñer, Prats y Braga (2009). El Síndrome se evaluó en 11530 profesionales hispanos, divididos en $51 \%$ varones y $49 \%$ mujeres, con una media de 41,7 años. El equipo de investigación aplicó el Maslach Burnout Inventory (MBI), compuesto por 22 ítems (con siete opciones de respuesta), divididos en tres dimensiones: agotamiento emocional, despersonalización y realización personal.

Los autores realizaron cruces entre países y profesiones. En términos generales las prevalencias más altas del Burnout correspondieron a los residentes de España, Argentina y Uruguay, con cifras entre 14,9\% y 7,9\%. Según la profesión, la prevalencia más alta correspondió a Medicina con 12,1\%, seguido de Enfermería y Odontología, mientras Psicología presentó un puntaje inferior a $6 \%$.
Algunas variables identificadas como protectoras fueron: la edad, el tener hijos(as), el sentirse valorado(a), el optimismo, la satisfacción laboral y la valoración económica.

El estudio anterior permitió estimar los niveles de prevalencia en el campo de la salud; sin embargo, la extrapolación al campo penitenciario es baja, pues las condiciones de trabajo y las características de la población varían sustancialmente. Por ello, tal y como fue señalado previamente, el objetivo general del estudio fue caracterizar la presencia del Síndrome del Burnout y el Engagement en profesionales de la administración penitenciaria.

En el campo educativo, Alvarado (2009) validó la escala Maslach Burnout Inventory, con 131 docentes de primer y segundo ciclo de escuelas cartaginesas, tanto rurales como urbanas. El instrumento estudiado fue una versión de Gil-Monte y Peiró (1997), esta consta de 22 reactivos con una escala de respuesta tipo Likert de 6 puntos; los componentes de la escala fueron: agotamiento emocional, despersonalización y realización personal en el trabajo.

Los resultados de un análisis factorial confirmatorio, reafirmaron la validez y la confiabilidad de las escalas de agotamiento emocional y realización personal; sin embargo, deben mejorarse los datos referentes a la despersonalización, ya que algunos ítems no se ubicaron en los factores esperados y el Coeficiente Alpha de Cronbach fue inferior a 0,60 .

En Alvarado (2009) se recomienda continuar aplicando la escala a nivel nacional y prestar atención a la dimensión de despersonalización, pues sus coeficientes deben ser ajustados a los puntajes recomendados: superiores a 0,70.

Por otro lado, Calvo y Serrano (2010) evaluaron el Síndrome del Burnout en 110 policías penitenciarios de la Dirección General de Adaptación Social, funcionarios de tres centros de atención institucional: de San José $(n=50)$, de Cartago $(n=19)$ y de la Biblioteca Dr. Gerardo Rodríguez Echeverría $(n=41)$. Las investigadoras exploraron mediante un cuestionario (el cual incluyó la versión española de 15 ítems del Maslach Burnout Inventory) la relación del Síndrome del Burnout con variables sociales, emocionales y laborales. 
Dicho síndrome está presente en un nivel alto con $44,50 \%$ de la muestra $y$ en un nivel bajo para el 55,50\%. En cuanto a las tres dimensiones del Síndrome, se presentó un alto nivel de agotamiento en los policías de los tres centros estudiados; el cinismo pese a ser bajo, fue reportado por la población investigada, mientras la ineficacia profesional presentó altos puntajes.

Sobre el mismo tema, Hernández, Fernández, Romas y Contador (2006), investigaron el Síndrome del Burnout en funcionarios de un centro penitenciario en Topas, Salamanca. Con la participación de 133 funcionarios(as) de vigilancia del centro penitenciario de un total de 300 (44\% del total), se aplicó el Maslach Burnout Inventory para evaluar tres dimensiones: cansancio emocional, despersonalización y realización personal.

Como hallazgos del estudio, los sujetos con 40 años o menos revelaron significativamente mayores actitudes en despersonalización que los sujetos de mayor edad. Pero los resultados no mostraron diferencias significativas en las dimensiones del MBI en función de la experiencia profesional.

El equipo de la presente investigación se ha centrado en el modelo teórico de trabajo del equipo de WoNT (Work and Organizational Network) Prevención Psicosocial del Departamento de Psicología Evolutiva, Educativa, Social y Metodológica de la Universitat Jaume. A continuación, se revisan los aspectos centrales de la propuesta teórica de la presente investigación.

El desarrollo del concepto de Burnout como fenómeno psicológico se originó en Estados Unidos con los trabajos de Maslach, a mediados de la década de 1970. El Burnout es un proceso generado como consecuencia del estrés laboral crónico, en el cual se combinan variables de carácter individual, social y organizacional. Es decir, una respuesta prolongada a estresores crónicos a nivel personal $y$ relacional en el trabajo, determinada a partir de las dimensiones conocidas como: agotamiento, cinismo e ineficacia profesional (Breso, Salanova y Schaufeli, 2007).

Otra definición es la ofrecida por Maslach, quien lo señala como "un síndrome psicológico de agotamiento emocional, despersonalización y reducida realización personal que puede ocurrir en individuos normales que trabajan con personas de alguna manera" (1993: 20-21). Una metáfora para representarlo es un estado de agotamiento similar a un fuego que se sofoca, una pérdida de energía, una llama que se extingue o una batería que se agota. En tanto, se plantea que las personas generalmente inician sus actividades laborales experimentando bienestar y satisfacción, pero posteriormente recaen en un importante decaimiento (Salanova y Llorens, 2008).

Según Fidalto (2006), este síndrome aún no se incluye dentro de los Manuales de Clasificación Diagnóstica reconocidos por la comunidad psicológica, tales como el DSM IV y el CIE 10. Además, se considera al Burnout consecuencia de la exposición a estresores laborales y la interacción del trabajador(a) con condiciones de riesgo, es decir, una consecuencia sobre la salud de la persona derivada directamente del trabajo.

Para Fidalto (2006), el Síndrome conforma una respuesta al estrés cuando fallan las estrategias funcionales de afrontamiento: los esfuerzos cognitivos $y$ conductuales constantemente cambiantes, desarrollados para manejar las demandas específicas externas y/o internas evaluadas como excedentes o desbordantes de los recursos individuales, lo cual no necesariamente implica situar el origen o la causa en un fallo de la estructura o en la funcionalidad psíquica del trabajador, sino más bien, en las demandas emocionales, fundamentalmente las que sobrepasan la capacidad y "tolerancia" del trabajador(a). Así, el Síndrome del Burnout se comporta como una variable mediadora entre el estrés percibido y sus consecuencias.

Breso et ál. (2007), han definido el Síndrome a partir de tres componentes:

1. Agotamiento a nivel personal: en este rubro se incluye la sensación de no poder dar más de sí mismo a nivel emocional.

2. Cinismo: este se manifiesta a nivel social y se compone de una actitud distante ante las labores por realizar, las personas receptoras del servicio y los compañeros(as) de trabajo. 
3. Ineficacia profesional: se presenta la sensación de no hacer adecuadamente las tareas y ser incompetente en el trabajo.

Las repercusiones del Síndrome del Burnout se expresan en una serie de consecuencias agrupadas por áreas (Salanova $y$ Llorens, 2008):

$\diamond \quad$ A nivel individual: se presenta el agotamiento, la fatiga crónica, el cansancio, la distancia mental, la ansiedad, la depresión, las quejas psicosomáticas, el incremento del uso de substancias psicoactivas, la generalización o el desbordamiento de la vida privada e incertidumbre sobre la propia capacidad para desempeñar el trabajo.

$\diamond \quad$ En el área laboral: se denota en insatisfacción, falta de compromiso organizacional e intención de abandonar la institución.

$\diamond \quad$ En el aspecto organizacional: se manifiesta en un incremento de la tasa de ausentismo y bajas laborales (incapacidades), disminución del desempeño en el trabajo y la falta de calidad en el servicio.

Respecto a la etiología, Salanova y Llorens (2008) proponen la existencia de dos procesos explicativos del desarrollo del Burnout.

El primero se refiere al proceso desarrollado en profesionales de ayuda, en quienes el Síndrome se desencadenaría debido a las demandas interpersonales implicadas en la atención con pacientes/clientes/usuarios quienes podrían llegar a consumirles emocionalmente. Para evitar distanciarse de la fuente originaria del malestar, se adoptaría la despersonalización como estrategia de afrontamiento, la cual llevaría en última instancia, a una reducida realización personal.

Desde la teoría del desequilibrio esfuerzos-resultados, esta situación se explica como consecuencia de una falta de reciprocidad o percepción de injusticia entre las inversiones $y$ resultados presentes en una interacción social (Salanova y Llorens, 2008).
El segundo proceso de desarrollo del Burnout sería más general y podría presentarse en cualquier profesional. Se asume que, independientemente de la ocupación, una falta de confianza en las propias competencias resulta un factor crítico en el desarrollo del Burnout.

Según investigaciones recientes, la presencia de altas demandas y pobres recursos generaría crisis sucesivas de auto-eficacia, las cuales con el transcurso del tiempo derivarían en el Síndrome del Burnout; específicamente en agotamiento, distancia mental (cinismo $y$ despersonalización) e ineficacia profesional (Llorens, García y Salanova, 2005; Salanova y Llorens, 2008).

El equipo de investigación planteó la medición y el contraste de dos constructos, el "Burnout" y el "Engagement". Este último, es definido como un constructo motivacional positivo relacionado con el trabajo, caracterizado por el vigor, la dedicación y la absorción; se refiere a un estado cognitivo-afectivo persistente en el tiempo y focalizado sobre un objetivo o conducta específica. Sus componentes son los siguientes:

1. El vigor se caracteriza por altos niveles de energía mientras se trabaja, de persistencia $y$ de un fuerte deseo de esforzarse en el trabajo.

2. La dedicación se manifiesta por altos niveles de significado del trabajo, entusiasmo, inspiración, orgullo y retos vinculados con la tarea por realizar.

3. La absorción se caracteriza por estar plenamente concentrado(a) y feliz realizando el trabajo mientras se tiene la sensación de que el tiempo pasa "volando" y la persona se deja llevar por el trabajo (Salanova y Llorens, 2008).

A continuación, se presenta el método utilizado en la investigación, los detalles del instrumento y el procesamiento de los datos.

\section{MÉTODO}

El presente estudio fue desarrollado con un diseño transversal o transaccional, es de tipo descriptivo y se adhiere al paradigma cuantitativo, por lo tanto, se privilegió la recolección 
de datos numéricos para explicar la relación entre variables previamente establecidas a partir de datos teóricos, es decir, el Síndrome del Burnout, el Engagement, las variables sociodemográficas y las laborales.

\section{PARTICIPANTES}

Los participantes son profesionales que laboran en la administración penitenciaria y los criterios de inclusión consistieron en: ser profesional en ciencias sociales, estar nombrado en algún puesto del Ministerio de Justicia y Paz, además de participar voluntariamente como sujeto de investigación.

La técnica de recolección de datos empleada fue la encuesta y se accedió a una muestra no probabilística (accidental o por conveniencia), es decir, a quienes cumplieron los criterios anteriores se les explicó el objetivo de la investigación y las condiciones para participar, por último, quienes brindaron su consentimiento verbal se les hizo completar un cuestionario autoaplicado.

En el estudio participaron 180 personas, distribuidas en las siguientes profesiones: Psicología, Orientación, Derecho, Trabajo Social y unos pocos de Criminología, Educación, Agronomía, Administración y Sociología.

\section{INSTRUMENTO}

El equipo de investigación desarrolló un cuestionario autoaplicado compuesto por 49 preguntas distribuidas en cuatro apartados. La primera sección, la conformó la presentación del estudio y el consentimiento informado.

En la segunda parte, se solicitó a las personas completar los datos personales y laborales, es decir, preguntas como el sexo, la edad, los años de trabajar en el Sistema Penitenciario, el puesto y la profesión.

La tercera parte, agrupó las escalas del Burnout y Engagement respectivamente, cada una conformada por 15 ítems con categorías de respuesta tipo Likert de siete puntos, el nivel de medición se entiende como: a mayor puntaje, mayor acuerdo con cada una de las afirmaciones propuestas.

La última parte del instrumento presentó interrogantes asociadas con el uso del tiempo libre, la jornada laboral y la intención de cambiar de empleo.

Se realizó un etapa de validación cualitativa del instrumento, es decir, se elaboró una primera versión que fue examinada por el equipo de investigación; seguidamente, se solicitó a dos varones $y$ a dos mujeres funcionarios(as) de la Dirección General de Adaptación Social, completar el cuestionario para recabar datos sobre la comprensión de las preguntas, la adecuación del lenguaje y la extensión del mismo. El tiempo de aplicación del instrumento osciló entre 10 y 15 minutos.

A partir de este ejercicio, se incluyeron tres preguntas y se ajustaron algunos términos por ser confusos, también se determinó conveniente la extensión del mismo y de esta manera, se obtuvo la versión final del cuestionario.

\section{CARACTERÍSTICAS PSICOMÉTRICAS DE LAS ESCALAS}

Los ítems de las escalas empleadas fueron debidamente recodificados para aplicar análisis de frecuencias y consistencia interna. Los reactivos de la escala del Engagement fueron sometidos a un análisis de consistencia interna, se calculó el Coeficiente Alpha de Cronbach y se obtuvo un puntaje de 0,88 . Las correlaciones ítem total se ubicaron entre 0,28 y 0,76 , lo cual señala la adecuada consistencia interna de la escala (ver la tabla 1).

La escala utilizada para estimar el nivel de Burnout igualmente fue sometida a un análisis de consistencia interna (ver la tabla 2), el resultado mostró un Coeficiente Alpha de Cronbach de 0,86, las correlaciones ítem total de los reactivos se ubicaron entre 0,11 y 0,76 . 
TABLA 1

RESULTADOS DEL ANÁLISIS DE CONSISTENCIA INTERNA DE LA ESCALA ENGAGEMENT

\begin{tabular}{lccc}
\hline & MEDIA & $D E$ & $r_{i t}$ \\
\hline Mi trabajo es retador & 4,91 & 1,43 & 0,47 \\
Mi trabajo me inspira & 4,82 & 1,24 & 0,72 \\
Estoy entusiasmado sobre mi trabajo & 4,71 & 1,33 & 0,76 \\
Estoy orgulloso del trabajo que hago & 5,29 & 1,01 & 0,61 \\
Mi trabajo está lleno de significado y propósito & 5,01 & 1,24 & 0,63 \\
En mi trabajo me siento lleno de energía & 4,25 & 1,40 & 0,63 \\
Puedo continuar trabajando durante largos periodos de tiempo & 3,96 & 1,62 & 0,52 \\
Cuando me levanto por las mañanas tengo ganas de ir a trabajar & 4,46 & 1,41 & 0,64 \\
Soy muy persistente en mi trabajo & 5,22 & 1,01 & 0,63 \\
Soy fuerte y vigoroso(a) en mi trabajo & 4,98 & 1,10 & 0,67 \\
Cuando estoy trabajando olvido todo lo que pasa alrededor de mí & 3,35 & 1,97 & 0,28 \\
El tiempo vuela cuando estoy trabajando & 4,74 & 1,47 & 0,48 \\
Me dejo llevar por mi trabajo & 3,95 & 1,59 & 0,51 \\
Estoy inmerso(a) en mi trabajo & 4,46 & 1,45 & 0,56 \\
Soy feliz cuando estoy absorto(a) en mi trabajo & 3,86 & 1,69 & 0,52 \\
\hline Alpha de Cronbach & & 0,88 & 153 \\
Número de sujetos & & 27 & \\
Valores perdidos & & & \\
\hline
\end{tabular}

Fuente: Elaboración propia.

TABLA 2

RESULTADOS DEL ANÁLISIS DE CONSISTENCIA INTERNA DE LA ESCALA BURNOUT

\begin{tabular}{|c|c|c|c|}
\hline & MEDIA & $D E$ & $r_{i t}$ \\
\hline Estoy emocionalmente agotado(a) por mi trabajo. & 2,77 & 1,55 & 0,68 \\
\hline Estoy muy cansado(a) al final de un día de trabajo. & 3,44 & 1,77 & 0,55 \\
\hline Estoy cansado(a) cuando me levanto en la mañana y tengo que afrontar otro día en mi puesto de trabajo. & 2,58 & 1,70 & 0,70 \\
\hline Trabajar todo el día es una tensión para mí. & 2,07 & 1,69 & 0,68 \\
\hline Estoy quemado(a) por mi trabajo. & 1,97 & 1,84 & 0,76 \\
\hline He perdido interés por mi trabajo desde que empecé en este puesto. & 0,98 & 1,33 & 0,62 \\
\hline He perdido entusiasmo por mi trabajo. & 1,10 & 1,27 & 0,75 \\
\hline Me he vuelto más cínico* respecto a la utilidad de mi trabajo. & 1,14 & 1,51 & 0,55 \\
\hline Dudo de la trascendencia y valor de mi trabajo. & 0,88 & 1,34 & 0,44 \\
\hline Puedo resolver de manera eficaz los problemas que surgen en mi trabajo. & 0,97 & 1,14 & 0,11 \\
\hline Estoy quemado(a) por mi trabajo. & 0,77 & 1,06 & 0,15 \\
\hline En mi opinión soy bueno(a) en mi puesto. & 0,43 & 0,68 & 0,31 \\
\hline Me estimula conseguir objetivos en mi trabajo. & 0,57 & 0,99 & 0,45 \\
\hline He conseguido muchas cosas valiosas en este puesto. & 1,22 & 1,41 & 0,29 \\
\hline En mi trabajo, tengo la seguridad de que soy eficaz en la finalización de las cosas. & 0,64 & 0,95 & 0,39 \\
\hline Alpha de Cronbach & & 0,86 & \\
\hline Número de sujetos & & 157 & \\
\hline Valores perdidos & & 23 & \\
\hline
\end{tabular}

Fuente: Elaboración propia. 


\section{PROCEDIMIENTO}

La aplicación de los cuestionarios se realizó durante reuniones programadas semestralmente por las diferentes jefaturas de los Departamentos, en dichos espacios, previa revisión de los criterios de inclusión, se invitó a los funcionarios(as) a participar en la investigación, por lo tanto, la muestra del estudio es no probabilística, con un muestreo a conveniencia que no permite la generalización de los resultados.

Un miembro del equipo de investigación permaneció cerca de los(as) informantes para responder preguntas. La aplicación de los instrumentos se efectuó durante junio y julio de 2009. Para garantizar la confidencialidad de los participantes, la aplicación y la recolección de los instrumentos fue anónima, una vez recibido el documento se depositó en un sobre para posteriormente digitarlo.

Una vez completa la recolección de cuestionarios, los datos se procesaron con el Paquete Estadístico para Ciencias Sociales (SPSS v. 18, por sus siglas en inglés). Posteriormente, se aplicaron análisis de frecuencias, análisis de contingencias, análisis de correlaciones, pruebas de hipótesis y análisis de consistencia interna. Seguidamente, se presentan los resultados de la investigación desarrollada.

\section{RESULTADOS}

\section{DESCRIPCIÓN DE LA MUESTRA}

En el estudio participaron 180 funciona-rios(as) de la administración penitenciaria, todos profesionales del campo de las ciencias sociales. Los participantes fueron 70\% $(n=126)$ mujeres y $30 \%(n=54)$ varones. La edad de los mismos se ubicó entre los 23 y los 61 años, con una media aritmética de 41,47 años ( $D E=10,51)$.

El estado civil de la mayor parte de los funcionarios(as) fue casado(a) (47,5\%), seguido por soltero(a) con $31,3 \%$, en tercer lugar divorciado(a) con $11,2 \%$, las personas que se encuentran en unión libre fueron 7,8\%, mientras el $1,1 \%$ es viudo(a) y 1,1\% separado(a). El $71,7 \%$ tiene hijos(as) y la cantidad de los mismos osciló entre 1 y 7, la media se ubicó en 2,1 hijos(as).
El lugar de residencia predominante fue la provincia de San José (46,1\%), se ubicaron en segundo y tercer lugar Alajuela y Heredia, mientras un porcentaje inferior al $10 \%$ residen en las provincias restantes.

Predominó la religión Católica con 68,4\%, seguida por la Evangélica con 15,80\%, una minoría aseguraron ser Gnósticos con $0,60 \%$ y Adventistas un $1,20 \%$, finalmente, $14 \%$ no practica ningún credo.

El tiempo de laborar en el Sistema Penitenciario es sumamente variable, pues el mínimo fue un mes y el máximo 40 años, la media correspondió a 14,1 años, con una desviación estándar de 11 años.

La muestra se distribuyó según la profesión de la siguiente manera: la mayor parte desempeñan la profesión de trabajadores(as) sociales $(35,6 \%)$, seguido por los abogados(as) $(26,1 \%)$, psicólogos(as) (21,7\%) y orientadores(as) (10,6\%), en una menor proporción se ubicaron las profesiones en Criminología, Sociología, Educación, Agronomía y Administración, los cuales ascienden a $6 \%$ en total.

El nivel académico de los participantes se distribuyó de la siguiente forma: Bachillerato universitario 9,6\%, Licenciatura 78,1\%, Maestría 10,7\%, egresada de Licenciatura 1\% y Doctorado $0,6 \%$. El nombramiento es interino para el $31,1 \%$ y en propiedad para $68,9 \%$.

Los programas en que laboran los informantes fueron los siguientes: $0,6 \%$ en la Dirección de Policía, el 1,1\% en las Oficinas Centrales, el $9,7 \%$ en el programa en Comunidad $y$ en el de Atención Penal Juvenil se ubicaron $10,3 \%$. El programa semi-institucional cuenta con una representación del 11,4\%. Finalmente, el 66,9\% de profesionales trabajan en el programa institucional, este corresponde a los Centros de Atención Institucional, conocidos como centros penales o propiamente "cárceles". Cabe destacar que un 72,5\% de los informantes han laborado en algún otro programa de la Dirección General de Adaptación Social.

\section{RESULTADOS SOBRE EL BURNOUT Y EL ENGAGEMENT}

Los datos de la tablas 1 y 2 señalan que la medición de los constructos de interés fue consistente en este grupo específico de 
profesionales. La información descriptiva de las escalas en estudio puede ser consultada en las tablas mencionadas; la primera, corresponde al Engagement y resalta los reactivos "Estoy orgulloso del trabajo que hago", pues puntuó 5,29 , la media más alta de los 15 reactivos propuestos. En el otro extremo, se ubicó: "Cuando estoy trabajando olvido todo lo que pasa alrededor de mi”, pues obtuvo la media más baja con 3,35; sin embargo, cabe señalar que ningún ítem se situó por debajo de 3 -la media teórica de esta escala-, lo cual indica puntajes altos para los ítems propuestos.

En la escala del Burnout, el ítem que mejor refleja niveles bajos es: "En mi opinión soy bueno(a) en mi puesto" con una media de 0,43, - después de la debida recodificación de las categorías de respuesta-, por otro lado, "Estoy muy cansado(a) al final de un día de trabajo" indicó alto Burnout con una media aritmética de 3,44, el puntaje más alto alcanzado por los informantes en esta escala.

Las escalas del Burnout y Engagement contaron con diversas puntuaciones, la cuales pueden ser revisadas en la tabla 3:

TABLA 3

ESTADÍSTICAS DE TENDENCIA CENTRAL Y DISPERSIÓN PARA LAS ESCALAS DEL ESTUDIO

\begin{tabular}{lcc}
\hline & $\begin{array}{c}\text { ESCALA } \\
\text { BURNOUT }\end{array}$ & $\begin{array}{c}\text { ESCALA } \\
\text { ENGAGEMENT }\end{array}$ \\
\hline Mínimo & 0 & 1,43 \\
Máximo & 5 & 6 \\
Media & 1,47 & 4,53 \\
Mediana & 1,34 & 4,60 \\
Moda & 1,00 & 5,07 \\
Desviación estándar & 0,88 & 0,89 \\
\hline
\end{tabular}

Fuente: Elaboración propia.
La media de la escala del Burnout fue 1,47 y la desviación estándar 0,88 . Si se considera como punto de referencia que la escala propuesta para medición contaba con siete opciones de respuesta, el puntaje reportado por los profesionales entrevistados es mínimo, aunque el dato indica la presencia del Síndrome en el grupo de trabajadores. De forma opuesta, en una escala con las mismas medidas, el Engagement puntuó 4,53, una cifra cerca de tres veces mayor que el Burnout reportado. La relevancia de los datos anteriores teóricamente se asociaría con puntuaciones altas en las diferentes dimensiones del constructo estudiado; sin embargo, los datos empíricos no siguen ese patrón.

Los datos estadísticos de las dos escalas fueron evaluados en los diferentes grupos de profesionales, en orden descendente la organización fue: abogados(as), trabajadores(as) sociales, psicólogos(as) y orientadores(as) para el Burnout, aunque las medias se diferenciaron entre el mínimo y máximo puntaje por únicamente en 0,33 puntos (ver tabla 4 ).

Los profesionales de Criminología, Sociología, Educación, Agronomía y Administración fueron excluidos del análisis por contar con menos de diez participantes, lo cual limitaba las posibilidades de cotejo.

Para profundizar aún más en las puntuaciones de cada uno de los subcomponentes de las escalas se confeccionó la tabla 5 , la cual contiene información sobre las medidas de tendencia central y dispersión de cada constructo (ver tabla 5).

En las subescalas del Engagement los puntajes mínimos fueron superiores a 0 , mientras en la escala del Burnout el resultado fue 0. Igualmente, en todas las escalas se alcanzaron los puntajes máximos, excepto en la subescala cinismo con 4,50. 
TABLA 4

ESTADÍSTICAS DE LAS ESCALAS DEL ESTUDIO SEGÚN LA PROFESIÓN

\begin{tabular}{cccc}
\hline PROFESIONES & MEDIDAS & ESCALA BURNOUT & ESCALA ENGAGEMENT \\
\hline Derecho & Media & 1,62 & 4,36 \\
$n=47$ & $D E$ & 1,06 & 0,99 \\
\hline Trabajo Social & Media & 1,50 & 4,62 \\
$n=64$ & $D E$ & 0,84 & 0,82 \\
\hline Psicología & Media & 1,43 & 4,33 \\
$n=39$ & $D E$ & 0,83 & 1,00 \\
\hline Orientación & Media & 1,29 & 4,66 \\
$n=19$ & $D E$ & 0,64 & 0,54 \\
\hline
\end{tabular}

Fuente: Elaboración propia.

TABLA 5

ESTADÍSTICAS DE TENDENCIA CENTRAL Y DISPERSIÓN PARA LAS SUBESCALAS DEL ESTUDIO

\begin{tabular}{lcccccc}
\hline & \multicolumn{2}{c}{ SUBCOMPONENTES DE BURNOUT } & \multicolumn{3}{c}{ SUBCOMPONENTES DE ENGAGEMENT } \\
\hline & AGOTAMIENTO & CINISMO & PROFESIONAL & DEDICACIÓN & VIGOR & ABSORCIÓN \\
\hline Mínimo & 0 & 0 & 0 & 0,40 & 1,50 & 0,4 \\
Máximo & 6 & 4,50 & 6 & 6 & 6 & 6 \\
Media & 2,57 & 0,80 & 1,09 & 4,93 & 4,55 & 4,12 \\
Mediana & 2,20 & 0,66 & 0,75 & 5,20 & 4,80 & 4,20 \\
Moda & 1 & 0 & 0 & 6 & 6 & 4,60 \\
Desviación estándar & 1,52 & 0,71 & 1,23 & 1,06 & 1,07 & 1,14 \\
\hline
\end{tabular}

Fuente: Elaboración propia.

Para determinar la proporción de personas que presentaron altos y bajos niveles del Síndrome $y$ la medición opuesta, las cifras se recodificaron en un nivel bajo y alto, para ello se utilizó la mediana teórica (tres para ambos casos).

Los datos de la tabla 6 indican que la proporción de personas con un alto nivel de
Burnout es de 5,6\%, mientras este grupo en la variable Engagement, aumentó a 94,4\%. Los puntajes son equivalentes para las variables $y$ corresponden a 10 sujetos. Ahora bien, debido a las actividades laborales desarrolladas por los informantes, se esperaba una mayor proporción de personas con alto nivel de Burnout,

TABLA 6

REPORTE DE NIVELES BAJOS Y ALTOS DE ENGAGEMENT Y BURNOUT

\begin{tabular}{ccccccc}
\hline & \multicolumn{2}{c}{ BAJO } & \multicolumn{2}{c}{ ALTO } & \multicolumn{2}{c}{ TOTAL } \\
\hline & $f$ & $\%$ & $f$ & $\%$ & $f$ & $\%$ \\
\hline Engagement & 10 & 5,6 & 170 & 94,4 & 180 & 100 \\
Burnout & 170 & 94,4 & 10 & 5,6 & 180 & 100 \\
\hline
\end{tabular}

Fuente: Elaboración propia. 
por lo tanto, los bajos puntajes reportados requieren un análisis más específico en futuras investigaciones.

Los promedios de cada subescala fueron agrupados de acuerdo a la media teórica (tres) y como dato relevante, las tres subdimensiones del Burnout se ubicaron por debajo de este puntaje, mientras las correspondientes al Engagement son superiores a cuatro en todos los casos. La desviación estándar de los seis aspectos revisados es baja, pero en comparación con la media, esta es alta para los constructos: cinismo e ineficacia.

De los datos anteriores, se concluye que la subvariable del Burnout más relevante en este grupo de profesionales fue el agotamiento, ya que las dos restantes presentaron puntuaciones muy bajas.

El porcentaje de personas con niveles altos y bajos en los factores evaluados se reporta en la tabla 7.

TABLA 7

ANÁLISIS DE DATOS SEGÚN NIVELES BAJO Y ALTO DE LAS DIMENSIONES DE CADA VARIABLE

\begin{tabular}{|c|c|c|c|c|c|}
\hline \multirow{2}{*}{ ESCALAS } & \multirow{2}{*}{ SUBESCALAS } & \multicolumn{2}{|c|}{ BAJO } & \multicolumn{2}{|c|}{ ALTO } \\
\hline & & $f$ & $\%$ & $f$ & $\%$ \\
\hline \multirow{3}{*}{ Burnout } & Agotamiento & 116 & 64,4 & 64 & 35,6 \\
\hline & Cinismo & 179 & 99,4 & 1 & 0,6 \\
\hline & Ineficacia profesional & 166 & 92,2 & 14 & 7,8 \\
\hline \multirow{3}{*}{ Engagement } & Dedicación & 13 & 7,2 & 167 & 92,8 \\
\hline & Vigor & 23 & 12,8 & 157 & 87,2 \\
\hline & Absorción & 32 & 17,8 & 148 & 82,2 \\
\hline
\end{tabular}

Fuente: Elaboración propia.

De la escala del Burnout, el constructo de mayor interés es el agotamiento, en tanto un $35,6 \%$ presentó un nivel alto frente $64,4 \%$ con puntajes bajos; en las dimensiones cinismo e ineficacia, las proporciones altas no superaron el 7,8\%.

En la escala del Engagement, los porcentajes de personas con un nivel alto son superiores a $82 \%$ en todos los casos. Sin embargo, porcentajes bajos entre $7,2 \%$ y $17,8 \%$ no son despreciables.

El Burnout y Engagement según niveles bajo $y$ alto, fueron analizados de acuerdo a la profesión; los resultados contenidos en la tabla 8 indican que el total de orientadores participantes presentó un bajo nivel de Burnout y un alto nivel de Engagement.

TABLA 8

ANÁLISIS DE DATOS SEGÚN NIVELES BAJO Y ALTO DE LAS VARIABLES POR PROFESIÓN

\begin{tabular}{|c|c|c|c|c|c|c|}
\hline \multirow{2}{*}{ PROFESIONES } & \multicolumn{3}{|c|}{ BURNOUT } & \multicolumn{3}{|c|}{ ENGAGEMENT } \\
\hline & NIVELES & $f$ & $\%$ & NIVELES & $f$ & $\%$ \\
\hline Orientador(a) & Bajo & 19 & 100 & Alto & 19 & 100 \\
\hline \multirow{2}{*}{ Psicólogo(a) } & Bajo & 37 & 94,9 & Bajo & 4 & 10,3 \\
\hline & Alto & 2 & 5,1 & Alto & 35 & 89,7 \\
\hline \multirow{2}{*}{ Abogado(a) } & Bajo & 43 & 91,5 & Bajo & 4 & 8,5 \\
\hline & Alto & 4 & 8,5 & Alto & 43 & 91,5 \\
\hline \multirow{2}{*}{ Trabajador(a) Social } & Bajo & 60 & 93,8 & Bajo & 2 & 3,1 \\
\hline & Alto & 4 & 6,3 & Alto & 62 & 96,9 \\
\hline
\end{tabular}

Fuente: Elaboración propia. 
Los profesionales en Psicología presentaron un nivel bajo de Burnout y alto de Engagement, con porcentajes inferiores a 6\% en el primero y superiores a $85 \%$ en el segundo; lo mismo sucedió con los(as) profesionales en Derecho y Trabajo Social (ver tabla 8).

La frecuencia absoluta de participantes con un bajo nivel del Burnout asciende a 10 y quienes presentaron un bajo nivel del Engagement coinciden en esta misma cifra.

Con el propósito de estimar la relación entre las variables teóricas propuestas y las variables laborales revisadas, se efectuó un análisis de correlación de Pearson, los datos con significancia estadística se reportan en la tabla 9.

Los años de laborar en el Sistema Penitenciario correlacionó únicamente con el agotamiento, siendo esta una relación positiva, lo cual significa que entre más años de trabajo se presenta mayor agotamiento $(r=0,16 ; p<$ $0,05)$. Pese a que la correlación es baja, es estadísticamente significativa y corresponde a un primer indicador asociado con el desarrollo del Burnout en este grupo de profesionales.

Las horas dedicadas a la recreación correlacionaron significativamente con tres variables: Burnout $(r=-0,30)$, agotamiento $(r=-$ $0,32)$ e ineficacia profesional $(r=-0,19)$. Es decir, entre más horas dedicadas a la recreación, la persona experimenta menor presencia de Burnout, menor agotamiento y menor ineficacia profesional; esta misma asociación se estableció positivamente con el vigor $(r=0,29)$. Lo anterior ubica a la recreación como una variable de interés, pues se relaciona positivamente con aspectos promotores del bienestar personal $y$ laboral en este grupo.

El Burnout y el Engagement presentaron una asociación negativa, moderada $y$ estadísticamente significativa, es decir, entre mayor Burnout experimentan los informantes menor Engagement $(r=-0,56, p<0,01)$ y viceversa.

El Burnout interactúa positivamente con las tres subescalas que lo componen. Asimismo, actúa negativamente con significancia estadística con los tres subcomponentes del Engagement (dedicación, vigor y absorción). Por lo tanto, los tres subcomponentes de cada escala correlacionan positiva $y$ negativamente según corresponda con las subescalas de la variable opuesta.

Una comparación de medias entre hombres y mujeres en las mediciones del Burnout y Engagement, indicaron la ausencia de diferencias grupales en esta muestra, la presencia del Síndrome es independiente del sexo. Sin embargo, es clave que las mujeres están sobrerrepresentadas en la muestra, al ser 126 y los varones únicamente 54.

Los informantes indicaron trabajar un mínimo de ocho horas a la semana y un máximo de 80 , lo cual supera la jornada máxima de 48 horas semanales. En contraste, las horas dedicadas a la recreación oscilaron entre cero $y$ un máximo de 80. Al mismo tiempo, 19 personas indicaron disfrutar cero horas para la recreación, 15 sujetos aseguraron contar con dos horas para la recreación y 18 personas señalaron contar con cinco horas a la semana, datos que si bien no son la mayoría, evidencian la desigual distribución entre la jornada laboral y el tiempo libre.

La media de horas laboradas por semana fue la esperada con 42, ello contrasta con la media de 8,59 horas por semana dedicadas a la recreación, con una moda entre cero y diez horas por semana (ver tabla 10). 

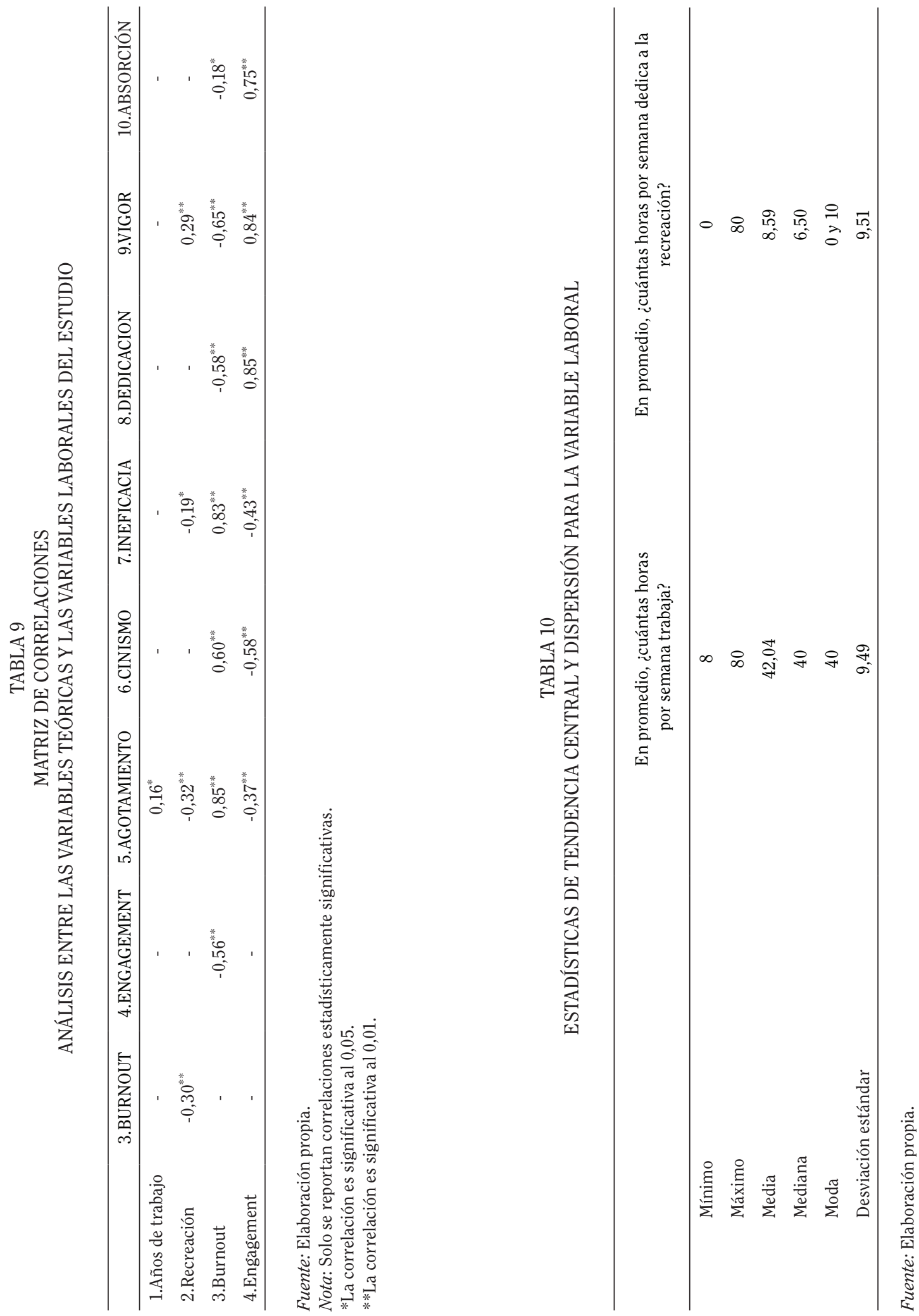

Rev. Ciencias Sociales Universidad de Costa Rica, 137: 65-81 / 2012 (III). (ISSN: 0482-5276) 
La tabla 11 presenta los resultados de un cruce entre las horas dedicadas a la recreación y los niveles de Burnout, siendo llamativa la acumulación entre 0 y 10 horas dedicadas al ocio por parte de los participantes.
Con el Engagement sucedió lo mismo, hay una concentración de personas que dedican entre 0 y 10 horas a la recreación en los niveles bajos y altos, pese a existir una correlación importante.

TABLA 11

HORAS DE RECREACIÓN POR NIVELES DE BURNOUT

\begin{tabular}{lcccc}
\hline & & \multicolumn{3}{c}{ HORAS DE RECREACIÓN } \\
\cline { 3 - 5 } & & ENTRE $0-10$ & ENTRE 11-20 & MÁS-20 \\
\hline \multirow{2}{*}{ Burnout } & Bajo & 110 & 30 & 30 \\
& Alto & 7 & 1 & 2 \\
\cline { 2 - 5 } & TOTAL & 117 & 31 & 32 \\
\hline \multirow{3}{*}{ Engagement } & Bajo & 5 & 1 & 4 \\
& Alto & 112 & 30 & 32 \\
\cline { 2 - 5 } & TOTAL & 117 & 31 & \\
\hline
\end{tabular}

Fuente: Elaboración propia.

Nota: No se reportan los valores perdidos.

Las horas dedicadas a la recreación no se vinculan —al menos en el análisis de contingencias anterior - con los niveles bajos o altos de Burnout, por lo cual, la relación debe explorarse más en otras investigaciones.

TABLA 12

DESCRIPCIÓN DE LOS RESULTADOS DE VARIABLES ASOCIADAS ANTIGÜEDAD, CONDICIONES DE TRABAJO E INTENCIÓN DE CAMBIAR DE TRABAJO

\begin{tabular}{lcc}
\hline & Sí & NO \\
\hline ¿El tiempo que usted labora es suficiente para cumplir con las tareas que se le asignan? & $38,8 \%$ & $61,2 \%$ \\
¿Cuenta con las condiciones necesarias para la ejecución de sus funciones? & $16 \%$ & $84 \%$ \\
¿Si usted tuviera la posibilidad de cambiarse a otro trabajo con el mismo salario, lo haría? & $44,4 \%$ & $55,6 \%$ \\
\hline
\end{tabular}

Fuente: Elaboración propia.

El 38,8\% de la muestra aseguró contar con el tiempo suficiente para cumplir con sus labores. Las condiciones de trabajo fueron valoradas como inadecuadas para el desarrollo de las labores por el $84 \%$ y como un indicador indirecto de la satisfacción laboral, el 44,4\% de los sujetos señaló que si tuviera la posibilidad de cambiar de trabajo con el mismo salario lo haría.
En la tabla 13, se registra la distribución de un análisis de contingencia con los resultados de la intención de cambiar de trabajo y los niveles bajo y alto de Burnout reportados por los profesionales evaluados (para determinar los niveles bajos $y$ altos se utilizó la media teórica de la escala $=3,5$ ). 
TABLA 13

INTENCIÓN DE CAMBIAR DE TRABAJO POR NIVELES DE BURNOUT

\begin{tabular}{ccccc}
\hline \multirow{2}{*}{ ESCALAS } & \multirow{2}{*}{ NIVELES } & \multicolumn{2}{c}{ CAMBIARIA DE TRABAJO } & \multirow{2}{*}{ TOTAL } \\
\cline { 2 - 4 } & & SÍ & NO & \\
\hline \multirow{2}{*}{ Burnout } & Bajo & 66 & 95 & 161 \\
& Alto & 10 & 0 & 10 \\
\cline { 2 - 4 } Engagement & TOTAL & 76 & 95 & 171 \\
\hline \multirow{3}{*}{ Bajo } & 9 & 1 & 10 \\
& Alto & 67 & 94 & 161 \\
\hline & TOTAL & 76 & 95 & 171 \\
\hline
\end{tabular}

Fuente: Elaboración propia.

Nota:ota. No se reportan los valores perdidos.

Las diez personas que presentan un nivel alto de Burnout estarían dispuestas a cambiar de trabajo, mientras quienes presentan un nivel bajo no muestran un patrón particular: 66 personas sí cambiarían y 95 personas no. La distribución es similar para el constructo Engagement, de las diez personas con bajos puntajes, nueve sí cambiarían de trabajo y una persona no lo haría. En síntesis, existe una relación entre los niveles de Burnout y Engagement, al mismo tiempo que la intención de cambiar la actividad laboral desarrollada.

En el siguiente apartado, se presentan las conclusiones y la discusión derivadas de los datos revisados en el apartado de resultados.

\section{CONCLUSIONES Y DISCUSIÓN}

Los datos revisados en el apartado anterior, permiten señalar varias conclusiones, las cuales se señalan a continuación.

Los 180 profesionales encuestados son un grupo disímil en cuanto a las características socio-demográficas, especialmente, las prácticas religiosas, la experiencia laboral y las condiciones familiares, con predominio de población femenina, lo cual es característico del campo de las ciencias sociales.

Con independencia de los resultados por variable, los datos psicométricos de las escalas indicados por los Coeficientes Alpha de Cronbach, señalan una medición confiable de los constructos en estudio, pues la asociación reportada fue la teóricamente esperada.
Prevaleció un bajo nivel de Burnout y un alto nivel de Engagement, lo cual podría ser explicado por la dispersión de las características del grupo, o bien, por condiciones particulares, por ejemplo: la reciente incorporación de profesionales al puesto de trabajo y por lo tanto, a la institución. Dentro de las subvariables de interés, el agotamiento merece especial atención, ya que impacta otras condiciones de las personas, tales como el tiempo dedicado a la recreación.

Tal y como se planteó teóricamente, quienes presentan altos niveles de Burnout estarían dispuestos a cambiar de trabajo; pero hay un patrón equivalente en quienes reportaron un alto nivel de Engagement, alrededor de lo cual surgen diferentes hipótesis, por ejemplo, quienes permanecen "motivados y absortos" en su trabajo, podrían contar con un bajo nivel de vinculación a la institución y más bien interesarse en el crecimiento laboral, independientemente del lugar.

Claramente existe una relación positiva entre el nivel de Burnout y la intención de abandonar la institución, lo mismo sucede con quienes presentan un bajo nivel de Engagament, las cuales estarían dispuestas a movilizarse a otras instituciones.

El Síndrome del Burnout y del Engagement se comportaron como variables relacionadas empíricamente: con coeficientes de correlación negativos y estadísticamente significativos; es decir, se puede impactar el 
Síndrome del Burnout tratando de modificar los niveles de Engagement y viceversa.

En otros estudios, variables como la edad, el sexo y la jornada laboral, han mostrado relación con la presencia del Síndrome, en este caso, una limitación para determinar posibles asociaciones de este tipo fue que la población femenina triplica la masculina. Para futuras investigaciones se recomienda estudiar la población por separado, es decir, investigar a los profesionales varones $y$ mujeres independientemente $y$ luego realizar comparaciones.

Son de especial atención las pocas horas dedicadas a la recreación, lo cual se torna más relevante, en tanto el uso del tiempo libre y la recreación contribuyen con el desempeño laboral y crecimiento personal, es decir, un grupo de personas significativo estaría expuesto a importantes niveles de estrés, por la ausencia de espacios para el esparcimiento $y$ la salud mental.

Las mediciones del Burnout y del Engagement deben continuar siendo realizadas en esta población y asociadas con otros datos como el uso del tiempo libre, pues si bien, se reportaron niveles que podrían requerir intervenciones individuales, posiblemente otros factores requieran intervenciones individuales o grupales.

Dentro de la medición de variables laborales, la antigüedad correlaciona de forma importante con el agotamiento $y$ negativamente con las horas dedicadas a la recreación, por lo tanto, la asociación entre estas variables requiere movilizar recursos institucionales para generar mecanismos compensatorios en personas con gran cantidad de tiempo de laborar. Debe recordarse que algunos informantes tenían más de 30 años de laborar para dicho Ministerio.

Es fundamental, meditar los resultados anteriores en función de la promoción de estilos de vida saludables y políticas de gestión del talento humano. De momento, temas como el uso del tiempo libre, el auto-cuidado, la salud mental $y$ otros aspectos promotores de la salud ocupacional, deben ser estudiados y posteriormente fortalecidos en este grupo de trabajadores.

\section{REFERENCIAS}

Alvarado, K. "Validez factorial de Maslach Burnout Inventory (versión castellana) en educadores costarricense". Actualidades Investigativas en Educación 9. 2009: 1-22.

Bianchini, M. "El Síndrome del Burnout en personal profesional de la salud". Revista de Medicina Legal de Costa Rica 13-14. 1997: 189-192.

Breso, E.; Salanova, M. y Schaufeli, W. "Síndrome de estar quemado por el trabajo 'Burnout' (III): instrumento de medición”. Notas técnicas de prevención. España: Instituto Nacional de Seguridad e Higiene en el Trabajo, 2007.

Calvo, K. y Serrano, D. "Síndrome de Burnout en policías penitenciarios de tres centros de atención institucional costarricenses". [Tesis para optar por el grado de Licenciatura en Psicología]. Universidad Fidélitas, 2010.

Cardenal, V. y Alonso, J. "Un estudio sobre la incidencia del Burnout entre los trabajadores del Centro Penitenciario de Huelva”. Apuntes en Psicología 23. 2005: 151-160.

Fidalgo, M. "Síndrome de estar quemado por el trabajo o 'Burnout' (I): definición y proceso de generación”. Notas técnicas de prevención. España: Instituto Asturiano de Prevención de Riesgos Laborales, 2006.

Grau, A.; Flichtentrei, D.; Suñer, R.; Prats, M. y Braga, F. "Influencia de los factores personales, profesionales $y$ transnacionales en el Síndrome del Burnout en personal sanitario hispanoamericano y español". Revista Española de Salud Pública 83 (2). 2009: 215-230.

Goffman, E. Relations in public: microstudies of the public order. London: Lane The Penguin Press, 1971.

Hernández, L.; Fernández, B.; Ramos, F. y Contador, I. "El Síndrome de Burnout en funcionarios de vigilancia de un centro penitenciario". International Journal of Clinical and Health Psychology 6. 2006: 599-611. 
Madrigal, M. "'Burnout' en médicos: aproximaciones teóricas y algunos diagnósticos diferenciales". Revista de Medicina Legal de Costa Rica 23. 2006: 137-153.

Porras, E. "Actividad laboral con privados de libertad". Revista de Medicina Legal de Costa Rica 13-14. 1997: 184-188.

Salanova, M. y Llorens, S. "Estado actual y retos futuros en el estudio del Burnout". Papeles del Psicólogo 29. 2008: 59-67.

Salanova, M.; Schaufeli, W.; Llorens, S.; Peiro, J. M. y Grau, R. "Desde el 'Burnout' al
'Engagement': ¿una nueva perspectiva? Revista de Psicologia del Trabajo y las Organizaciones 16. 2000: 117-134.

OTROS

Dirección General de Adaptación Social (Costa Rica). Ley 4762. Asamblea Legislativa, 1971.

Fecha de ingreso: $22 / 07 / 2011$ Fecha de aprobación: 18/01/2012 
